


\section{Article Artículo Paper Los Servicios Sociales como 3 marco organizacional para la participación de las familias}

\section{Josep Maria Torralba ${ }^{1}$}

María Virginia

Matulic $^{2}$

Marta Arranz

Xavier Canals ${ }^{4}$

\section{Resumen}

Los Servicios Sociales como Sexto Sistema de Protección Social del Estado del Bienestar es el marco organizacional donde se atienen y acompañan las personas usuarias y las familias desde los Servicios Básicos de Atención Social (en adelante, SBAS) de la ciudad de Barcelona. Es el contexto institucional y la dimensión organizativa que hace posible la acción de los profesionales en la atención a las necesidades sociales de las personas usuarias y las familias, así como su participación en este proceso.

El artículo que se presenta se enmarca en la primera fase de la investigación participativa [Re] Pensamos la participación de las familias: Diagnóstico y propuestas de intervención en los Servicios de Atención Básica de las personas en la ciudad de Barcelona (20192022) realizada por el Grupo de Investigación e Innovación en Trabajo Social (GRITS) de la Universitat de Barcelona. El diseño de la investigación es de tipo

1, 2, 3, 4. Profesores/as y miembros del Grupo de Investigación e Innovación en Trabajo Social (GRITS), Unidad de Formación e Investigación-Escuela de Trabajo Social, Universitat de Barcelona. 
cualitativo, con un enfoque de investigación-participativa en que están reconocidos todos los actores/as. Para la recogida de los datos, se han utilizado las herramientas de la entrevista individual y el grupo de discusión.

Los resultados del estudio se presentan a partir de las categorías de las estrategias de participación desde la perspectiva local, las adaptaciones metodológicas para favorecer la participación, y el rol y responsabilidad de los agentes del sistema. Para finalizar, se argumentan las principales conclusiones de la investigación y se formulan algunas propuestas de mejora de la participación de las familias en los servicios sociales. Se constata la necesidad de implementar metodologías participativas en los SBAS a todos los niveles de intervención individual y familiar, grupal y comunitaria. Y que para construir ciudades inclusivas, es necesario implementar políticas centradas en la creación de vínculos sociales.

Palabras clave: Servicios sociales básicos de atención social, participación de las familias, marco organizacional, rol y responsabilidad de los agentes, metodologías participativas, vínculos sociales.

\section{Abstract}

Social Services as the Sixth Social Protection System of the Welfare State is the organizational framework in which users and families of the Basic Social Care Services (SBAS) of the city of Barcelona are attended and accompanied. It is the institutional context and the organizational dimension that makes possible the action of the professionals in the attention to the social needs of the users and the families, as well as their participation in this process.

The article presented is part of the first phase of participatory research [Re] We think about the participation of families: Diagnosis and intervention proposals in the Basic Attention Services of people in the city of Barcelona (2019-2022) carried out by the Group for Research and Innovation in Social Work (GRITS) of the University of Barcelona. The research design is qualitative, with a participatory-research approach in which all the actors are recognized. For data collection, the tools of the individual interview and the discussion group have been used. 
The results of the study are presented from the categories of participation strategies from the local perspective, the methodological adaptations to favor participation, and the role and responsibility of the agents of the system. Finally, the main conclusions of the research are argued and some proposals are made to improve the participation of families in social services. The need to implement participatory methodologies in SBAS is confirmed at all levels of individual and family, group and community intervention. In addition, that to build inclusive cities, it is necessary to implement policies focused on creating social ties.

Key words: Basic social care services, family participation, organizational framework, role and responsibility of agents, participatory methodologies, social ties. 


\section{Introducción.}

La complejidad de los hechos sociales y las situaciones de dificultad en las cuales se encuentran las personas usuarias de servicios sociales requieren de compromisos, competencias e interacciones entre los distintos actores sociales, siendo la participación un elemento consustancial en el contexto social en el cual nos encontramos. La participación en los Servicios Sociales Básicos (en adelante, SSB) es un aspecto transversal o de corresponsabilidad en el modelo de gestión e intervención, dado que es un cambio de posición de los profesionales, instituciones, ciudadanos y entidades sociales ante los nuevos contextos. Favorecer espacios de escucha y dar voz tanto a las personas usuarias como a los profesionales genera oportunidades para conocer las necesidades reales de la población (Pastor, 2009).

La participación de la ciudadanía en las decisiones que les afectan es la base de la democracia participativa. Y para que esta sea real y efectiva, es fundamental que la información y la capacidad de decisión puedan propiciar una relación horizontal entre la Administración y los ciudadanos/as. Con este artículo pretendemos reflexionar sobre la importancia de desarrollar y evaluar acciones de participación activa de las personas usuarias y las familias en los servicios sociales como verdaderos ciudadanos/as que aportan su visión y vivencias en los procesos de intervención profesional y sobre la toma de decisiones en los diversos itinerarios de atención social.

El artículo que se presenta se enmarca en las dos primeras fases de la investigación participativa [Re]Pensamos la participación de las familias: Diagnóstico y propuestas de intervención en los Servicios de Atención Básica de las personas en la ciudad de Barcelona (20192022) realizada por el Grupo de Investigación e Innovación en Trabajo Social (GRITS) de la Universitat de Barcelona, cuyo objetivo es construir un diagnóstico y propuestas de cambio, colectivamente, desde las miradas de los distintos actores sobre la participación de las familias en los itinerarios de atención en los servicios sociales de atención básica.

Diferentes estudios evidencian que la participación de la ciudadanía en los proyectos de investigación, desde una perspectiva de investigación participativa, es una excelente vía para acceder a grupos de población de difícil acceso (Newell y South, 2009). También que los enfoques de investigación participativa, tal y como señala Beresford (2013), reducen la separación entre los profesionales, la política y los usuarios de los 
servicios, como consecuencia del desafío y la ruptura del "nosotros" y "ellos ", a la vez que se suman conocimientos.

Otro foco, que se relaciona estrechamente con los objetivos de esta investigación, es el que señala Beresford (2005) cuando apunta que no se puede comprender la participación de las personas usuarias de los servicios en la investigación, sino se examina y se comprende antes la participación de éstas en la práctica. Algunos estudios en el contexto de los servicios sociales como el de Jenssen (2014), muestran experiencias colaborativas de éxito entre personas usuarias de servicios sociales, profesionales e investigadores. Otros estudios se enfocan en la evaluación colaborativa de funciones y competencias profesionales, en los que se destacan notables resultados en la mejora de la capacitación profesional fruto de la retroalimentación de las personas atendidas (Di Bailey, 2005). Las perspectivas de investigación participativa sobre las prácticas de intervención social se transforman, en consecuencia, en instrumentos acordes con los principios subyacentes en los códigos éticos profesionales y en los principios que enmarcan las leyes de servicios sociales, posibilitando procesos de conocimiento compartidos (Barbera, 2008).

El artículo está estructurado en los siguientes apartados: esta introducción sobre el propósito del artículo y el estado de la cuestión sobre la temática objeto de estudio; un segundo apartado sobre el diseño metodológico de la investigación; a continuación, un tercero sobre el análisis de resultados y discusión de las distintas categorías analizadas; y, por último, un quinto apartado con las conclusiones y las propuestas de mejora para finalizar con las referencias bibliográficas.

\section{Diseño metodológico}

Este estudio, de carácter cualitativo, ha contado con la participación directa de 126 personas, mediante las técnicas de la entrevista individual y de los grupos de discusión, distribuidos entre profesionales que ocupaban un cargo de gestión en la estructura de los servicios sociales del Ayuntamiento de Barcelona, profesionales involucrados en la atención social básica y personas usuarias o familias vinculadas a los servicios sociales. El trabajo de campo se ha desarrollado entre los meses de diciembre del 2019 a enero del 2020. 
Con respecto a las entrevistas, se han mantenido un total de 38 entrevistas, de las cuales 9 han sido a cargos de gestión, 15 a profesionales y 14 a familias. En cuanto a los grupos de discusión, se han realizado un total de 9 grupos de discusión, con la participación de un total de 87 personas, distribuidos entre 2 grupos para cargos de gestión, 3 para profesionales y 4 para familias tal y como se muestra en la siguiente Tabla 1.

Tabla 1. Número de participantes y técnicas.

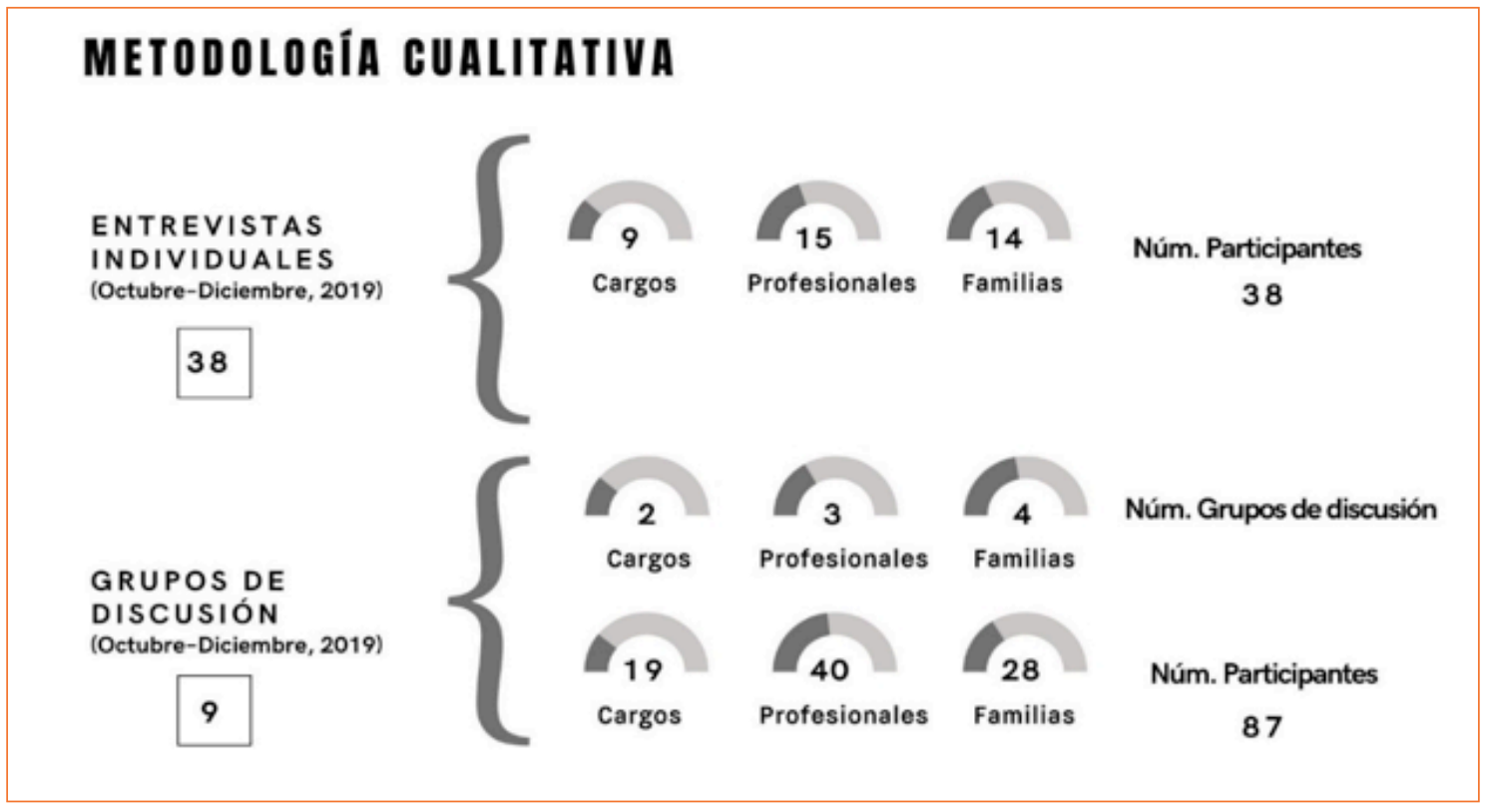

Fuente: Elaboración propia.

Para el análisis de la información, se han transcrito las entrevistas y los grupos de discusión, y se han elaborado los códigos para realizar la codificación de los datos mediante el programa Atlas.ti v.8.

\section{Análisis de resultados y discusión.}

En este apartado, se presentan los resultados obtenidos de la investigación así como su discusión a partir de las distintas categorías analizadas. 
a) Estrategias de participación desde la perspectiva local.

La participación es un proceso inmerso en la dinámica de la relación entre los profesionales y las personas usuarias de servicios sociales tal y como se muestra en el siguiente fragmento de entrevista: "Participar es cuando la persona o la familia está realmente implicada en su proceso de cambio". (E-profesional)

La década de los años 90 fue un periodo de importantes transformaciones organizativas y de nuevas líneas participativas generadas por un empowerment político local. Los ayuntamientos desarrollan a través de la proximidad, las herramientas y procesos de participación con sus comunidades de referencia (Blanco y Gomà, 2002). A partir del siglo XXI los gobiernos locales articulan varias estrategias y sistemas que permiten una participación más extensa de la ciudadanía (Díaz, 2017). La Ley 57/2003, de Medidas para la Modernización del Gobierno Local, trató de fijar unos estándares mínimos de participación en los municipios que introducían órganos como el Consejo social de ciudad, de carácter consultivo para el desarrollo local y la planificación urbana; la Comisión de sugerencias y reclamaciones para la defensa de los derechos de los vecinos; y órganos de gestión desconcentrada como los distritos (Corchete, 2015).

La participación "implica dar tu opinión o tu visión de determinadas cosas, compartir cosas con los otros, y participar para llegar a un acuerdo". (E-profesional). También dependerá de las circunstancias y de las características de cada persona, así como de los sentimientos y emociones que genera dirigirse a servicios sociales tal y como verbaliza una persona usuaria "cuando voy a servicios sociales, encima que estoy pidiendo no me puedo quejar, me tengo que conformar con el que me dan" (E-familia).

E1 Ayuntamiento de Barcelona (2017) aprobó un nuevo Reglamento de participación ciudadana donde se definían los canales de participación, los recursos necesarios y un sistema de garantías que velase por la democracia y su buen funcionamiento. En la actualidad se produce una institucionalización de la participación ciudadana en las políticas, procesos y programas de los gobiernos municipales que se reflejan en la producción de sistemas estables de participación (Parara y Resende, 2009). Y se configura de esta forma un modelo de gobierno local relacional que plantea la gestión municipal en términos de proximidad, implicación y deliberación conjunta con la ciudadanía (Blanco y Gomà, 2002). 
Entre las estrategias de participación desarrolladas por los gobiernos locales, hay que mencionar los consejos, las comisiones, las mesas y las redes. Estos órganos de participación tienen varias funciones que se analizan a continuación.

\section{Los consejos}

Los gobiernos locales disponen de órganos de participación que se establecen como canales de encuentro y de interlocución entre la ciudadanía y el ayuntamiento. En función de su ámbito pueden ser órganos territoriales (de ciudad, distrito o barrio) y órganos sectoriales que están relacionados con una temática concreta que pueden ser de ciudad o distrito (Ayuntamiento de Barcelona, 2019). Los órganos territoriales son aquellos que tienen como objetivo la mejora de un determinado ámbito geográfico. Se dispone de varios niveles como sueño el consejo de ciudad, los consejos ciudadanos de distrito y los consejos de barrio.

El consejo de ciudad es el máximo órgano consultivo y de participación en el cual los representantes del ayuntamiento y la ciudadanía debaten los asuntos principales de la ciudad. Las actividades se presentan en un informe anual donde el gobierno municipal rinde cuentas de todas las actividades realizadas.

Los consejos de distrito se rigen según el Reglamento de organización y funcionamiento de los distritos y tienen funciones de asesoramiento y sostén de los consejos sectoriales y consejos de barrio. Y estos últimos son órganos de participación y de fortalecimiento comunitario creados para abordar todas las cuestiones relativas al barrio.

\section{Comisiones y Mesas}

Las comisiones pueden ser impulsadas por el ayuntamiento o determinadas entidades, y tienen como objetivo promover y desarrollar proyectos de mejora concretos. Las mesas pueden ser territoriales como órganos consultivos que tienen el objetivo de coordinar, impulsar y promover políticas sobre temáticas que afectan al territorio; y de coordinación como instrumentos de gobernanza municipal para trabajar de forma conjunta, administración y entidades, problemáticas que afectan la ciudadanía (Jardín et al., 2016).

\section{Las redes}

Las redes son espacios de participación y acción conjunta entre instituciones y organizaciones de la ciudad para cooperar y tejer acciones compartidas en temas co- 
munes que inciden en el bienestar de las personas. Varios autores centran como bases fundamentales el trabajo en red, la cooperación, la reciprocidad y la solidaridad (Rovere, 1999; Ubieto, 2007).

Desde el año 2006, se articula en la ciudad de Barcelona el Acuerdo Ciudadano por una Barcelona Inclusiva que reúne además de 700 entidades sociales. En este espacio, se concreta la dimensión relacional de la acción de gobierno en materia de política pública de inclusión en el marco del Plan Municipal para la Inclusión Social 2005-2010.

En la actualidad, este acuerdo se encuentra en proceso de desarrollo de la Estrategia de inclusión y de reducción de las desigualdades sociales en la ciudad. La finalidad es elaborar el Plan de Acción e impulsar la coproducción entre áreas municipales, entidades del Tercer Sector Social, movimientos sociales y redes de acción para promover la inclusión social y reducir las desigualdades sociales en los próximos diez años.

Las redes de acción nacen con la voluntad de trabajar conjuntamente sobre un mismo eje y/o materia y lograr mejoras en los respectivos campos de acción. Actualmente existen 13 redes con varias temáticas de trabajo.

\section{b) Adaptaciones metodológicas para favorecer la participación.}

Las importantes transformaciones sociales y económicas en la actualidad están configurando nuevos escenarios cada vez más diversos y complejos que nos hablan de un cambio de época (Subirats, 2011). El impacto de la crisis genera un aumento de la demanda a los servicios sociales que incrementa sus competencias en los territorios de referencia (Matulic y Fustier, 2008). El modelo de atención no se ha adaptado a los cambios emergentes manteniendo una atención residual ante situaciones complejas que requieren de otras formas de intervención.

Las profesionales entrevistadas consideran que con los años ha evolucionado la manera de participar tal y como se ejemplifica en la siguiente citación de entrevista:

Creo que ha ido cambiando, no ha sido siempre igual. Antes se hacia mucho trabajo en el despacho, y ahora como que nos hemos dado cuenta que tenemos que hacer trabajo comu- 
nitario, trabajo grupal, que tenemos que implicar mucho más a las familias en el plan de trabajo. (E-profesional)

Uno de los elementos que ha cambiado es el lenguaje tal y como se muestra en el siguiente fragmento de entrevista: "Claro nosotros ya no los llamamos usuarios, los llamamos participantes" (E-profesional). No obstante, la participación en las entidades pequeñas del Tercer Sector Social se puede llevar a cabo con más facilidad que por ejemplo en los servicios sociales a causa de la sobrecarga del servicio.

Estos cambios requieren de nuevas políticas públicas que respondan a las necesidades plurales desde un enfoque integral. Tal como afirma Subirats (2011, p.14), “las políticas sociales de nueva generación deben orientarse a la equidad, valorizando la diversidad y apostando para fomentar procesos de empoderamiento individual y comunitario". Se hace necesaria la articulación de metodologías participativas que centren la atención en la creación y el fortalecimiento de los vínculos sociales para consolidar una ciudadanía inclusiva (De la Red y Barranco, 2014).

Actualmente, encontramos a profesionales implicados y convencidos en la participación tal y como se ilustra en la siguiente citación de entrevista:

Las formas de participación ideales se trazan a través de la transparencia ante las personas, la sinceridad por ambas partes, la confidencialidad, la confianza, y todo esto solo se consigue a partir de crear un vinculo con la persona. (E-profesional)

Los SSB como dispositivos de proximidad se encuentran en una posición privilegia$\mathrm{da}$, dado que se convierten en verdaderos observatorios de las desigualdades sociales y en una pieza clave en el despliegue de acciones participativas en los territorios de referencia (Matulic, 2004). Los municipios tendrían que incorporar un cambio en su rol de intermediación entre las organizaciones, la ciudadanía y los agentes sociales a través de lógicas basadas en la coproducción que fomenten la articulación de propuestas compartidas a favor del bien común (De la Red y Barranco, 2014; Parara et al., 2015).

Los resultados de la investigación ponen de relieve la necesidad de implementar metodologías participativas en todos los niveles de intervención, individual y familiar, grupal y comunitario tal y como se ejemplifica en la siguiente citación de entrevista: 
Yo creo que habría que buscar en las metodologias que ya existen de co-diseño, de solucionas entre usuarios y profesionales de los servicios. Quizás pensar al aplicar una metodología adaptada a este tipo de situaciones a las que nos enfrentamos, a qué tipo de acción queremos quitar a cabo. (EI-profesional)

En relación con el plan de intervención, las profesionales entrevistadas indican que intentan construirlo con la persona usuaria y su familia tal y como se ilustra en el siguiente fragmento de entrevista: "Se construye con el usuario y la familia, es decir no lo construyo yo aqui en el despacho, yo voy con la hoja blanca. Si él no participa de esto, son mis objetivos, no los suyos". También se pregunta "temporalidad, ¿̇y esto como lo quieres hacer?, ¿Con cuánto tiempo?, ¿Con dos meses?, ¿̇tres meses?”. (E-profesional)

La implicación activa de las familias en los procesos de intervención se produce a través de metodologías que incorporen diálogos apreciativos donde la intervención se construye de forma igualitaria y en cooperación con las personas (Renes et al., 2007). Desde esta perspectiva de co-construcción de saberes y de acción conjunta, se producen acompañamientos orientados a la autonomía y el empoderamiento individual y comunitario (Fernández et al., 2016).

\section{Recuperar la comunidad como espacio de intervención.}

Los escenarios complejos están impactando de manera directa en las familias más vulnerables, agravando las desigualdades sociales y debilitando los espacios de convivencia y cohesión social. Para construir ciudades inclusivas, es necesario implementar políticas centradas en la creación de vínculos sociales, donde están implicados profesionales, poderes públicos, entidades y ciudadanía (Subirats, 2004).

Los SSB de la ciudad de Barcelona están incorporando la perspectiva comunitaria en los territorios de referencia para trabajar colectivamente el empoderamiento y el fortalecimiento de las relaciones democráticas entre los diversos colectivos (Cerrajero y Morales, 2015). Estas estrategias rompen con el enfoque centrado en los recursos y los servicios, y de este modo construir otras formas de relación e implicación de las familias en sus propios procesos de reconstrucción individual y colectiva. 


\section{Potenciar las metodologías grupales y comunitarias.}

El despliegue del Nuevo modelo de SSB de la ciudad de Barcelona se produjo en el año 2008 con el objetivo de redefinir la cartera de servicios y prestaciones, promocionando metodologías más participativas en los barrios de la ciudad, entre las que se encuentran el trabajo grupal y comunitario (Ayuntamiento de Barcelona, 2015). Se incorpora una mirada comunitaria en los distintos niveles de intervención que introduce una visión colectiva de los problemas sociales en estos servicios sociales de proximidad (Cerrajero y Morales, 2015). Se señalan las ventajas de los proyectos grupales y comunitarios como generadores de redes de apoyo y cooperación entre las personas.

Los profesionales destacan como principal limitación la sobrecarga del trabajo directo que los impide poner en marcha nuevos proyectos participativos. A través del trabajo comunitario, se promueven espacios más horizontales y de empoderamiento de las personas que aporta beneficios a todos los actores implicados en el proceso de construcción colectiva (Cortés, 2014).

c) Rol y responsabilidad de los agentes del sistema.

Los agentes clave que interactúan en los SBAS son las personas usuarias, los profesionales y los cargos de gestión. El rol de los agentes en relación con la participación es distinto según cómo entienden la participación, cuál es su nivel de motivación y el grado de implicación, y cuál es el nivel de responsabilidad que tiene cada uno de ellos.

Se valora que el funcionamiento de los servicios sociales como marco organizacional puede condicionar las dinámicas de participación, así como las diferentes iniciativas de promoción de la participación de las familias, limitando los proyectos de mejora de la participación, tomando conciencia de estas limitaciones e intentando buscar alternativas tal y como se muestra en el siguiente fragmento de entrevista:

Yo no puedo llevar a cabo un proceso de participación y no poder decidir qué horario de servicios sociales tendremos, porque por mucho que vayamos a participar a pesar de decidir qué horario hay de centro, no es posible porque hay un marco que dice que en Barcelona Ciudad se atiende de tal hora a tal hora y tantos dias. (EI- Cargo de gestión) 
Esta reflexión de un cargo de gestión responde a las limitaciones que muy a menudo establece el marco institucional de los centros de servicios sociales y a la que los profesionales se deben enfrentar para desarrollar proyectos de dinamización y mejora de la participación de las familias en los servicios sociales. Muy a menudo los espacios dentro de los centros de servicios sociales no están preparados para poder realizar intervenciones que no sean solo entrevistas individuales, y esto no favorece las intervenciones de tipo grupal o más comunitarias (Retama et al., 2017). Tampoco se garantizan unos horarios de atención flexible para las personas usuarias y con una franja horaria más amplia que facilite estas intervenciones (Tabla de Entidades del Tercer Sector Social de Cataluña, 2016).

Tanto en las familias como en los profesionales, se identifica que el rol de las personas usuarias y de las familias respecto a la participación está relacionado con el nivel de vinculación establecido con los profesionales, así como con los proyectos de intervención grupal o bien con el plan de trabajo individual en que están involucrados tal y como se ejemplifica en la siguiente citación de entrevista:

El apoyo y la proximidad de la trabajadora social ha estado para mi mi salvación en todos los aspectos porque no sabía dónde tenía que ir, ahora pase el que pase después ya no es un tema que dependa de mi trabajadora social que hace el máximo para mi en cada momento. (EI- Familia)

Esta vivencia que manifiestan las familias es muy importante que quede recogida para dar el sentido a la intervención de los profesionales y de los principios de proximidad y accesibilidad que tendrían que velarse en todo momento en las distintas etapas de intervención con las personas usuarias en los diferentes itinerarios de atención social. En cualquier intervención profesional, es muy importante generar un buen vínculo entre profesionales y personas atendidas, y crear un clima de empatía, confianza e implicación entre estos (Ferrer, 2017; Pirla et al., 2019).

Algunos cargos de gestión reconocen que utilizar metodologías grupales con las personas usuarias y las familias genera resistencias entre los profesionales, a pesar de haber recibido formación sobre estas metodologías. El concepto ideal que se tiene desde el marco organizacional no siempre se ve acompañado en la práctica profesional, dado que el profesional tiene que salir de su zona de confort habitual como agente interviniente. 
Muy a menudo, los profesionales no se sienten bastante competentes para implementar nuevos proyectos de intervención grupal con las familias, o bien no quieren asumir el reto de experimentar nuevas metodologías de aproximación a las personas usuarias por miedo al fracaso. Y también no reciben el apoyo y reconocimiento necesario por parte de los cargos de gestión que impulse claramente la apuesta por estas nuevas metodologías de intervención. Es importante velar para que los profesionales de los servicios sociales salgan de su inmovilismo, y se consiga una mayor predisposición, interés y flexibilidad de estos para trabajar desde otros espacios de intervención (Ballester, 2015).

Tanto para los profesionales como por los cargos de gestión, está claro que las personas usuarias y las familias son corresponsables de su proceso de mejora junto con la intervención de los profesionales, pero que esto implica asumir cierto nivel de responsabilidad.

Lograr cierto nivel de responsabilidad y compromiso de las familias en su plan de intervención por parte de los profesionales implica muchas veces uno de los retos más complejos en su proceso de intervención, y al mismo tiempo es una forma de acompañamiento y apoyo fundamental con las personas usuarias en las distintas fases de esta intervención. Empoderar a una persona usuaria no quiere decir que la persona siga todas las indicaciones profesionales, sino que considere que estas forman parte de su vida y que se encuentran dentro de su voluntad de transformar la situación en la que vive (Pirla et al., 2019).

Tanto la carencia de formación como la pérdida de control por parte de los profesionales son dos factores que pueden limitar la generación de espacios participativos con las personas usuarias y las familias. El hecho de fomentar la participación en los servicios sociales puede comportar una percepción en los profesionales de carencia de control de la situación y también que se sientan menos competentes ante las nuevas dinámicas participativas que se generan tal y como se ejemplifica en el siguiente fragmento de entrevista: "Cuando tú pides participación a las personas usuarias y las familias, el profesional tiene que tener muy claro y estar dispuesto a perder cierto control dentro del marco de los servicios sociales." (EI- Profesional)

Esta reflexión de una profesional constata la necesaria formación continuada de los profesionales de los servicios sociales en las distintas metodologías de acción participativa de las familias, y el apoyo imprescindible de los cargos de gestión en la promoción y facilitación para dar respuesta a las diferentes necesidades formativas de los profesionales 
que refuercen sus competencias profesionales y la superación de sus posibles miedos ante los nuevos retos de la intervención profesional con las personas usuarias en el marco de los servicios sociales. Es muy necesario revisar la formación y capacitación de los profesionales para adaptarla a las nuevas metodologías de trabajo. Y por parte de los cargos de gestión es fundamental que se dé una convicción y una decisión firme al dar respuesta a esta necesidad (Ferrer, 2017).

\section{Conclusiones y propuestas de mejora.}

La participación en los SSB es un aspecto transversal o de corresponsabilidad en el modelo de gestión e intervención, dado que es un cambio de posición de los profesionales, instituciones, ciudadanos y entidades sociales ante los nuevos contextos.

El Gobierno de la Generalitat de Catalunya ha aprobado recientemente el II PESS 2021-2024, en el cual se apuesta por la participación de las personas usuarias y de los profesionales como una estrategia para fortalecer un sistema centrado en las personas y los profesionales, y se establece la necesidad de promover la participación de la ciudadanía en la definición y evaluación de las políticas sociales.

También el Ayuntamiento de Barcelona (2017) aprobó un nuevo Reglamento de participación ciudadana donde se definen los canales de participación, los recursos necesarios y un sistema de garantías que velase por la democracia y su buen funcionamiento. E1 despliegue del Nuevo modelo de SSB de la ciudad de Barcelona se produjo en 2008 con el objetivo de redefinir la cartera de servicios y prestaciones, promocionando y configurando metodologías más participativas en los barrios de la ciudad.

Los resultados de la investigación realizada ponen de relieve la necesidad de implementar metodologías participativas en el SBAS en todos los niveles de intervención, individual y familiar, grupal y comunitario. Y para construir ciudades inclusivas, es necesario implementar políticas centradas en la creación de vínculos sociales.

Los agentes clave que interactúan en los SBAS son las personas usuarias, los profesionales y los cargos de gestión. Cada proceso de participación puede ser particular en 
función de los agentes que interviene y de las dinámicas que se generan entre ellos. Se considera que el posicionamiento tanto de los profesionales como de los cargos de gestión influye en la promoción de la participación.

Los cargos de gestión y profesionales entrevistados valoran que el funcionamiento de los servicios sociales como marco organizacional puede condicionar las dinámicas de participación, así como las diferentes iniciativas de promoción de la participación de las familias. Tanto entre las familias como entre los profesionales, se identifica que el rol de las personas usuarias y de las familias respecto a la participación está relacionado con el nivel de vinculación establecido con los profesionales, así como con los proyectos de intervención grupal o bien con el plan de trabajo individual en que están involucrados.

Se valora que el encargo institucional que reciben los cargos de gestión y los profesionales que intervienen puede suponer una barrera para fomentar la participación de algunos grupos y familias. Y la carencia de formación, así como la pérdida de control por parte de los profesionales son dos factores que pueden limitar la generación de espacios participativos con las personas usuarias y las familias.

Como propuestas de mejora, se formulan las siguientes:

- Promover la implementación de proyectos de intervención grupal y comunitaria que favorezcan la participación real de las familias en los SBAS, integrando la opinión y la experiencia de las personas usuarias para mejorar los servicios sociales y que los perciban más cercanos;

- Facilitar a los profesionales de los SBAS los apoyos, habilidades, herramientas de trabajo y estructuras adecuadas para desarrollar sus competencias con suficiente capacidad de respuesta ante las nuevas necesidades sociales complejas y cambiantes de las familias y su atención y promoción social;

- Impulsar la investigación y la generación de conocimiento en la mejora e innovación de los SBAS con el fin de identificar y visibilizar buenas prácticas y nuevas metodologías de intervención social con las familias y personas usuarias con la participación de los colegios profesionales, las entidades del Tercer Sector Social y el mundo local. 


\section{Referencias bibliográficas}

Ajuntamentde Barcelona.(2005).Pla Municipalpera la Inclusió Social2005-2010.Barcelona: Regidoria de Benestar Social. http://www.bcn.cat/barcelonainclusiva/ca/2011/6/plainclusio.pdf

Ajuntament de Barcelona. (2015). Bases del model d'atenció grupal als serveis socials bàsics de l'Ajuntament de Barcelona: Conceptes, estratègies i eines per a la gestió. Barcelona: Regidoria de Qualitat de vida, Igualtat i Esports.

https://ajuntament.barcelona.cat/dretssocials/sites/default/files/arxius-documents/2015-bases-model-atencio-grupal-acc.pdf

Ajuntament de Barcelona. (2017). Reglament de participació ciutadana.

https://ajuntament.barcelona.cat/participaciociutadana/sites/default/files/documents/reglament_participacio_catala.pdf

Ajuntament de Barcelona. (2019). Guias prácticas de la participación ciudadana en Barcelona. Los órganos de participación.

https://ajuntament.barcelona.cat/participaciociutadana/sites/default/files/documents/2_esp_organs_orgcc.pdf

Ajuntament de Barcelona. (2020). L'Acord Ciutadà per una Barcelona inclusiva. http://www.bcn.cat/barcelonainclusiva/ca/estrategiainclusio.html

Ballester, M. (2015). Intervenció comunitària i rol dels professionals als serveis socials a Barcelona (Catalunya) i Mont-real (Quebec): una anàlisi comparada. [Tesis doctoral, Universitat de Barcelona]. Base de dades Tesis en Xarxa. https://www.tesisenred.net/ handle/10803/390949\#page=1

Barbera, R.A. (2008). Relationships and the Research Process: Participatory Action Research and Social Work. Journal of Progressive Human Services, (19)2, 140-159.

Beresford, P. (2005). Service user’: regressive or liberatory terminology? Disability E' Society, 20, 469- 477. 
Beresford, P. (2013). From 'other' to involved: user involvement in research: an emergint paradigm. Nordic Social Work Research, (3)2, 139-148.

Blanco, I., Gomà, R. (2002). Gobiernos Locales y Redes Participativas. Barcelona: Ariel.

Catalunya. (2007). Llei 12/2007, d'11 d'octubre, de serveis socials. (DOGC, núm. 4990, 11 octubre 2007, p. 37788-37839).

Corchete, M.J. (2015). Participación ciudadana en los asuntos públicos y su contribución a la calidad de la democracia. Reflexiones al bilo de algunas experiencias participativas. Bilbao: Universidad de Deusto.

Cortés, F. (2014). “La potenciació del treball comunitari com a estratègia per reafirmar el compromís social del treball social”. Revista de Treball Social (RTS), 203, 23-35.

De la Red, N.,Barranco, C. (2014). “Trabajo Social y participación en las políticas sociales". Azarbe, Revista Internacional de Trabajo Social y Bienestar, 3, 39-45.

Di Bailey, D. (2005). Using an action research approach to involving service users in the assessment of professional competence. European Journal of Social Work, (8)2, 165-179.

Díaz, A. (2017). “Participación ciudadana en la gestión y en las políticas públicas”. Gestión y Politica Pública, 26 (2), 341-379.

España. Ley 57/2003, de medidas para la modernización del gobierno local. (BOE, núm. 301, 17 diciembre 2003, p.44771-44791).

Fernández,J. (coord.). (2016). La intervenció amb families des del Treball Social. Grup de Recerca i Innovació en Treball Social (GRITS), Departament de Treball Social i Serveis Socials, Universitat de Barcelona. Barcelona: Col.legi Oficial de Treball Social de Catalunya. https://www.tscat.cat/content/la-intervencio-amb-les-families-des-del-treball-social

Ferrer, N. (2017). "Una aproximació a l'estat actual dels Serveis Socials. Estratègies pràctiques per millorar les intervencions socioeducatives en el Servei Bàsic d'Atenció Social". Educació Social. Revista d'Intervenció Socioeducativa, 65, 156-173. 
Generalitat de Catalunya. (2021). II Pla Estratègic de Serveis Socials 2021-2024. Barcelona: Departament de Treball, Afers Socials i Famílies. https://treballiaferssocials.gencat. cat/web/.content/03ambits_tematics/15serveissocials/pla_estrategic_serveis_socials/Pla_estrategic_serveis_socials_catalunya_NOU/01_Plana_principal/1.-202012-29-Pla-estrategic-de-serveis-socials-2021-2024.pdf

Ginesta, M., Civit, M., Rivera, J., Rodríguez, A. (2017). Dictamen: Els Serveis Socials Bàsics a la provincia de Barcelona. Situació actual i propostes de millora. Una mirada des del Treball Social. Barcelona: Col.legi Oficial de Treball Social de Catalunya, Monogràfic 13. https://www.tscat.cat/content/els-servesi-socials-basics-la-provincia-de-barcelona

Jardí, A., Parera,G., Serra, L. (2016). Guia metodològica per a la creació de taules de coordinació municipal entre Administracions i entitats. Barcelona: Diputació de Barcelona.

Jenssen, A.G. (2014). User involvement in practice research. Nordic Social Work Research, 4(1), 120-133

Manyà, C., Morales, E. (2015). Marc d'intervenció comunitària als Centres de Serveis Socials de Barcelona. Institut de Govern i Polítiques Públiques (IGOP).Bellaterra: Universitat Autònoma de Barcelona.

Matulic, M.V. (2004). "El reto pendiente del siglo XXI: Los servicios sociales de proximidad". Revista de Trabajo Social (RTS), 176, 57-69.

Matulic, M.V., Fustier, N. (2008). “Los servicios sociales básicos en la nueva ley de servicios sociales de Cataluña”. Revista de Servicios Sociales y Politica Social (83), 127-135.

Newell, C. J. \& South, J. (2009). Participating in community research: exploring the experiences of lay researchers in Bradford Community. WorkEFFamily, 12(1), 75-89.

Parés, M., Resende, P. (2009). Tipos de prácticas y experiencias de participación promovidas por la administración pública: Sus límites y radicalidades. En: M. Parés (coord.), Participación y calidad democrática: Evaluando las nuevas formas de democracia participativa, Barcelona: Ariel, p. 77-100. 
Parés, M., Castellà, C., García, M. (2015). Repensant la ciutadania la món local. Col·lecció documents de treball, 21. Barcelona: Diputació de Barcelona.

Pastor, E. (2009). “Participación y democratización de las políticas de servicios sociales en el ámbito municipal”. Portularia, (1), 69-81.

Pirla, A.,Julià, R.,Bertran, L.,Farré, A.,Haro, L.,Ibarz, I.,Massana, M.,Piñol, A.M.,Siscart, E.,Vilas, V.,Villafranca, A. (2019). La intervenció social amb famílies en situació de cronicitat en els Serveis bàsics d'atenció social. Barcelona: Col.legi Oficial de Treball Social de Catalunya, VIII Premi de Recerca Dolors Arteman. https://www.tscat.cat/publicacio/ viii-premi-dolors-arteman-millor-proposta-de-recerca-realitzar

Renes, V., Fuentes. P., Ruiz, E., Jaraíz, G. (2007). "Realidad, pensamiento e intervención social”. Documentación social, 145, 11-36.

Rovere, M. (1999). Redes en salud; un nuevo paradigma para el abordaje de las organizaciones y la comunidad. Rosario: Secretaría de Salud Pública/AMR, Instituto Lazarte.

Subirats, J. (2004). ¿Nuevas vías de participación ciudadana en los gobiernos locales? En: La Ley de Modernización del Gobierno Local. Barcelona: Fundació Carles Pi i Sunyer d'Estudis Autonòmics i Locals (Estudis 17, pp. 39-46).

Subirats, J. (2011). “Crisi o canvi d’època? Els impactes socials”. Revista de Treball Social (RTS), 194, 9-20.

Taula d'Entitats del Tercer Sector Social de Catalunya. (2016). La millora dels Serveis Socials Bàsics a Catalunya. Una proposta des del Tercer Sector Social. Debats Catalunya Social núm. 49. https://www.tercersector.cat/sites/default/files/dossier_serveis_socials.pdf

Ubieto, J. R. (2007). “Models de treball en xarxa”. Educació Social. Revista d'Intervenció Socioeducativa, 36, 26-39. 
